\title{
Surface modification of neural recording electrodes with conducting polymer/biomolecule blends
}

\author{
Xinyan Cui, ${ }^{1}$ Valerie A. Lee, ${ }^{2}$ Yehoash Raphael, ${ }^{2}$ James A. Wiler, ${ }^{2}$ Jamille F. Hetke, ${ }^{3}$ David J. Anderson, ${ }^{3}$ \\ David C. Martin ${ }^{1,4,5}$ \\ ${ }^{1}$ Macromolecular Science and Engineering Center, The University of Michigan, Ann Arbor, Michigan 48109 \\ ${ }^{2}$ Kresge Hearing Research Institute, Department of Otolaryngology, The University of Michigan, \\ Ann Arbor, Michigan 48109-0648 \\ ${ }^{3}$ Department of Electrical Engineering and Computer Science, The University of Michigan, \\ Ann Arbor, Michigan 48109-2122 \\ ${ }^{4}$ Department of Materials Science and Engineering, The University of Michigan, College of Engineering, 2022 H.H. \\ Dow Building, 2300 Hayward Street, Ann Arbor, Michigan 48109-2136 \\ ${ }^{5}$ Department of Biomedical Engineering, The University of Michigan, Ann Arbor, Michigan 48109-2136
}

Received 17 October 2000; revised 30 January 2001; accepted 16 February 2001

\begin{abstract}
The interface between micromachined neural microelectrodes and neural tissue plays an important role in chronic in vivo recording. Electrochemical polymerization was used to optimize the surface of the metal electrode sites. Electrically conductive polymers (polypyrrole) combined with biomolecules having cell adhesion functionality were deposited with great precision onto microelectrode sites of neural probes. The biomolecules used were a silk-like polymer having fibronectin fragments (SLPF) and nonapeptide CDPGYIGSR. The existence of protein polymers and peptides in the coatings was confirmed by reflective microfocusing Fourier transform infrared spectroscopy (FTIR). The morphology of the coating was rough and fuzzy, providing a high density of bioactive sites for interaction with neural cells. This high interfacial area also helped to lower the impedance of the electrode site and, consequently, to improve the signal transport. Impedance spectroscopy showed a lowered magnitude and phase of impedance around the biologically relevant frequency of $1 \mathrm{kHz}$. Cyclic voltammetry
\end{abstract}

demonstrated the intrinsic redox reaction of the doped polypyrrole and the increased charge capacity of the coated electrodes. Rat glial cells and human neuroblastoma cells were seeded and cultured on neural probes with coated and uncoated electrodes. Glial cells appeared to attach better to polypyrrole/SLPF-coated electrodes than to uncoated gold electrodes. Neuroblastoma cells grew preferentially on and around the polypyrrole/CDPGYIGSR-coated electrode sites while the polypyrrole $/ \mathrm{CH}_{3} \mathrm{COO}^{-}$-coated sites on the same probe did not show a preferential attraction to the cells. These results indicate that we can adjust the chemical composition, morphology, electronic transport, and bioactivity of polymer coatings on electrode surfaces on a multichannel micromachined neural probe by controlling electrochemical deposition conditions. (C) 2001 John Wiley \& Sons, Inc. J Biomed Mater Res 56: 261-272, 2001

Key words: conducting polymers; surface modification; neural microelectrode; cell attachment

\section{INTRODUCTION}

Micromachined neural prosthetic devices facilitate the functional stimulation of and recording from the peripheral and central nervous system. However, when these devices are implanted into brain tissue for

Correspondence to: D. C. Martin at Department of Materials Science and Engineering; e-mail: milty@umich.edu

Contract grant sponsor: NIH/NCRR; contract grant number: P41-RR09754, to The University of Michigan Center of Neural Communication Technology (CNCT)

Contract grant sponsor: NIH; contract grant number: NIDCD P01 DC00078

Contract grant sponsor: Sloan Foundation Fellowship

Contract grant sponsor: NSF; contract grant number: DMR-0084304

(c) 2001 John Wiley \& Sons, Inc. long-term recording, they lose electrical connectivity as a function of time. ${ }^{1,2}$ To solve this problem, the interfacial contact between the electrode sites and the biologic tissue must be improved.

Neurons, glia, and other tissue components will surround the implanted neural probe. With appropriate surface modifications, it may be possible to get selected neurons to attach or send processes directly onto the microelectrodes. A strong, mechanically intimate connection between the electrode surface and the neurons may prevent the formation of a nonconductive, organic boundary layer that presumably, in the long term, is involved in diminished functional activity. ${ }^{3}$ Work has been done on surface modification of silicon-based neural probes by electrospinning nanometer-diameter filaments of protein polymers. 
The enhanced attachment and growth of cells on the probe in vitro and in vivo has been demonstrated. ${ }^{4,5}$ However, this method does not have site specificity; in other words, the materials coat the entire probe. The overall biocompatibility of the probe may be improved, but the signal transport between the electrodes and the nervous system may not be enhanced by this approach.

To improve the signal transport at the electrode/ tissue interface, surface modification needs to be done right at the electrode sites. The electrode sites, which are flat or slightly concave metal or iridium oxide pads, are placed on the surface of the silicon-based probe. The ideal approach would (1) provide a more intimate contact between tissue and the microelectrode sites; (2) provide a much more highly effective interfacial area to enhance charge transport; and (3) provide biologic factors to attract neurons or neuronal processes onto the electrode site. We have developed an electrochemical mechanism that deposits the conducting polymer polypyrrole in combination with bioactive species onto microelectrode sites. Using this mechanism we have had promising results in acute in vivo recording and in vitro cell culture tests.

Polypyrrole is an electrically conductive polymer that can be polymerized electrochemically and deposited onto electrodes. The ease of preparation, inherent electrical conductivity, controllability of surface properties, and compatibility with mammalian cells make polypyrrole an attractive candidate for biomedical applications. ${ }^{6}$ Additionally, when polypyrrole is in its oxidized state, it exists as a polycation with delocalized positive charges along its conjugated backbone. In order to neutralize this charge, counter ions in the solution are incorporated into the polypyrrole film during electrochemical deposition. The conductivity, morphology, and stability of polypyrrole vary significantly when the counter ions are changed. ${ }^{7,8}$ When a polyelectrolyte having negative charges serves as the counter ion, it is incorporated into the polymer through the following reaction,

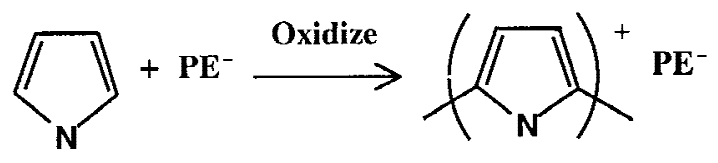

where $\mathrm{PE}^{-}$stands for polyelectrolyte. Based on this mechanism, bioactive species, such as DNA, heparin, poly(hyaluronic acid), proteins, nerve growth factors, and even red blood cells have been patterned onto electrode sites together with the electrically conductive polypyrrole. ${ }^{9-12}$

In the developing and maturing central nervous system (CNS), laminin plays a crucial role in cell mi- gration, differentiation, and axonal growth. ${ }^{13,14}$ It has been used extensively as a substrate for studies of the growth of nerve cells in vitro. ${ }^{15}$ It has been shown that partial functions of the large laminin molecule can be mimicked by small fragments, the sequences of which have been determined and localized. Such peptides include RGD, SIKVAV, YIGSR, and CDPGYIGSR. ${ }^{16-20}$ RGD has been found in most extracellular proteins, as well as in laminin, and has the function of promoting cell adhesion. ${ }^{16}$ The nonapeptide CDPGYIGSR is a newly discovered laminin fragment that has been proven to mediate cell attachment and migration and to enhance neurite extension from embryonic chick dorsal root ganglions. ${ }^{19,20}$ For the purpose of improving the cell-biomaterial interface, peptides and ECM proteins have been immobilized onto solid substrates such as glass, silicon micromachined probes, and glassy carbon electrodes. Various methods have been used, such as electron spinning, covalent bonding, chemisorption, self-assembling monolayers (SAM), microcontact printing, and electrochemical polymerization. ${ }^{4,5,21-25}$

We believe that electrochemical polymerization has many advantages over other methods in the surface modification of bioelectronic devices. The method can be used on many different electrically conductive surfaces whereas chemical attachment techniques usually are limited to a few substrate choices. The coating can be precisely patterned specifically onto the active site of the electrode without covering the nonfunctional areas of the device. The deposition of organic and biologic species does not compromise the electrical functionality of the electrode since the coating material is electrically conductive. On the contrary, we expect that the signal transport can be enhanced because of the unique surface morphology of the coatings. Huber and coworkers covalently linked various peptides with electropolymerizable monomer 3-hydroxyphenylacetic acid and then, through electropolymerization, immobilized derivatized peptides onto glassy carbon electrodes. ${ }^{25}$ In vitro cell culture tests showed a selective growth of cells on the CDPGYIGSR and 18-mer SIKVAV-coated electrode area. Nerve cell attachment on implanted glassy carbon pins coated with derivatized CDPGYIGSR was observed. They also compared the electrochemical method with adsorption and covalent coupling and found that peptides immobilized using electrochemical deposition demonstrated the highest efficiency.

In this paper, data will be presented on depositing polypyrrole combined with synthetic protein polymer SLPF and laminin fragment CDPGYIGSR. SLPF is a genetically engineered protein polymer with a major composition of GAGAGS sequences of silk alternated with the cell-binding sequence RGD. ${ }^{26}$ The nonapeptide CDPGYIGSR, as described above, promotes attachment and growth of cells in the nervous system. 
Unlike Huber's work, using the mechanism of Equation (1), we blended peptide and protein polymer into electrically conductive polymers during the electrochemical polymerization. This method does not require a special chemical coupling reaction; therefore it is easier to handle, safer in that it conserves bioactivity, and it provides more flexibility in the selection of bioactive species. As we demonstrate, in addition to peptide fragments, large proteins also can be blended in. The surface morphology, chemical composition, electrical activity, and in vitro cell culture results are shown below.

\section{MATERIALS AND METHODS}

\section{Silicon probes and material preparation}

Micromachined silicon probes were provided by the University of Michigan Center for Neural Communication Technology. A typical probe design is shown in Figure 1. The micromachined silicon substrate supports an array of thinfilm conductors that are insulated above and below by deposited dielectrics of silicon dioxide and silicon nitride. Openings in the upper dielectrics along the probe define the vertical connection to stimulating or recording sites that are sputtered with gold or iridium over the region of the top dielectrics for interfacing to the tissue. At the rear of the probe, the gold bond pads are present to connect with offchip instrumentation..$^{27,28}$ Gold was chosen as the electrode material in this work, rather than iridium, because it is stable under the electrochemical condition used during the polymerization while iridium tends to form various oxides.

Pyrrole monomer (99\%) was purchased from Aldrich. The silk-like polymer SLPF (ProNectin FO) was provided by Protein Polymer Technologies, Inc. The nonapeptide CDPGYIGSR was purchased from Sigma. All other routine chemical reagents were obtained from Aldrich.

\section{Modification of SLPF}

To accomplish the electrochemical deposition of polypyrrole/biomolecules from aqueous solution, the protein poly- mer SLPF had to be modified to enhance its water solubility and acidity. The modification used follows the modification procedures discussed by Stedronsky. ${ }^{29}$ Solubilization was achieved by reacting amine and hydroxyl groups in the protein polymer with propane sulfone to form bulky, anionic sulfopropyl groups. This method introduces additional polar functionalities to the highly repetitive protein polymer in order to disrupt the order of the protein, which then becomes soluble to water.

Specifically, SLPF (103 mg) and $3.0 \mathrm{~mL}$ of 4.5 molar aqueous lithium perchlorate were added to a $30-\mathrm{mL}$ jar with a rubber cap and agitated by a magnetic stirrer. The headspace was purged with nitrogen gas, and stirring started at ambient temperature. Propane sulfone (0.545 g; Aldrich), dissolved in $2.0 \mathrm{~mL}$ of tetrahydrofuran, was added in one portion to yield a homogeneous mixture. A solution $(1 \mathrm{~mL})$ of sodium hydroxide $(40 \mathrm{mg})$ dissolved in 4.5 molar of aqueous lithium perchlorate then was added by syringe at a rate of $20 \mu \mathrm{L} / \mathrm{min}$. After stirring for an additional $60 \mathrm{~min}$, acetic acid was used to adjust $\mathrm{pH}$ to $6.0-6.5$, and the reaction mixture was transferred to a $13 \mathrm{kDa}$ cutoff dialysis cellulose membrane (Membrane Filtration Products, Inc.) and dialyzed against $15 \mathrm{~L}$ of deionized water for $24 \mathrm{~h}$. The water was replaced and dialysis was continued for an additional $24 \mathrm{~h}$. The protein concentration of the solution was estimated as $4.68 \mathrm{mg} / \mathrm{mL}$ by UV spectroscopy.

\section{Electrochemical deposition}

Polypyrrole/bioactive molecule blends were grown galvanostatically on gold electrode sites $\left(1250\right.$ or $3900 \mu \mathrm{m}^{2}$ in area) of a neural probe at a current density of $0.5 \mathrm{~mA} / \mathrm{cm}^{2}$ (for SLPF) and $2 \mathrm{~mA} / \mathrm{cm}^{2}$ (for CDPGYIGSR). The current supply was a Potentiostat/Galvanostat EG\&G 283, through which a charge passed during the electrochemical reaction can be monitored. The electrochemical cell was a 3-mL vial soldered with a platinum wire as the counter electrode. The monomer solution $(1 \mathrm{~mL})$, which contained $0.3 \mathrm{M}$ of pyrrole and $4 \mathrm{mg} / \mathrm{mL}$ of solubilized SLPF or $1 \mathrm{mg} / \mathrm{mL}$ of CDPGYIGSR, was purged with $\mathrm{N}_{2}$ for approximately 5 min before use. The amount of material deposited onto the electrode was controlled by the charge passed during the deposition. For comparison, some electrodes were coated with polypyrrole doped with polystyrenesulfonate (PSS), $\mathrm{ClO}_{4}^{-}$, and

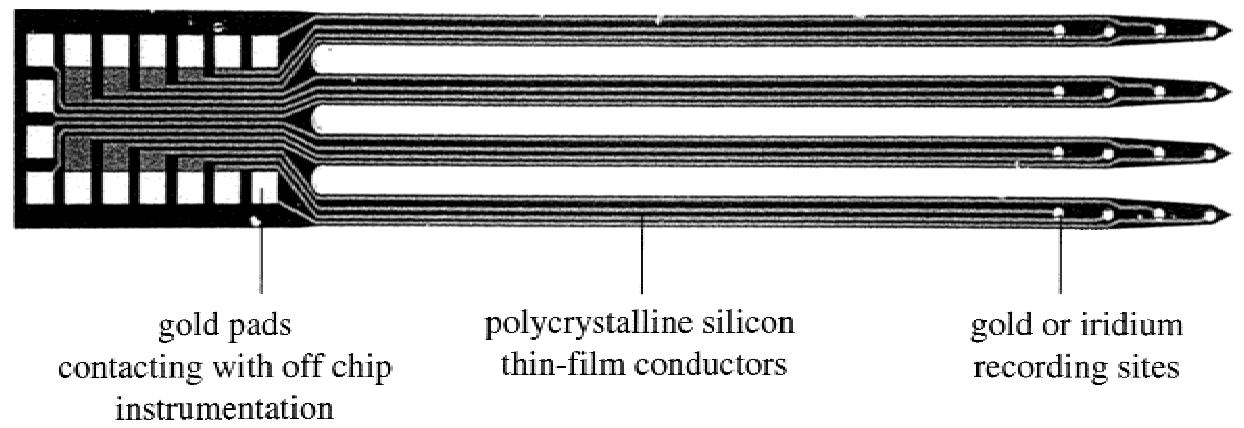

Figure 1. Micromachined silicon-based neural recording probe. This is a 4-shank, 16-channel recording electrode array. The thickness of the probe is about $15 \mu \mathrm{m}$. Features of the probes, including shank, site number, and spacing, can be customized to a given application. For these studies, the electrode site area varied from 177 to $3900 \mu \mathrm{m}^{2}$, and the distance between electrodes varied from 50 to $200 \mu \mathrm{m}$. 
$\mathrm{CH}_{3} \mathrm{COO}^{-}$. The experimental setup and procedure were almost the same as above except that the concentration of anion was $0.1 \mathrm{M}$.

\section{Microfocusing FTIR}

To characterize the chemical composition of the polypyrrole/biomolecule coatings, reflective Fourier transform infrared (FTIR) spectroscopy was performed using a PerkinElmer Spectrum GX attached to a PerkinElmer Multiscope equipped with a liquid-nitrogen-cooled MCT detector. The microscope accessory enabled the focusing of the IR beam on a small area of the sample. The microscope aperture was used to adjust the infrared light spot area. For a $1250-\mu m^{2}$ electrode, the aperture area was set at $36 \times 36 \mu \mathrm{m}^{2}$. A probe sample was placed on a gold mirror from which the background reflection signal was collected. The samples were scanned 100 times in the range of 4000 to $750 \mathrm{~cm}^{-1}$. PerkinElmer's Spectrum software, version 3.01, was used to generate the FTIR spectrum.

\section{Impedance spectroscopy measurements}

The instrument used for this study was an EG\&G electrochemical impedance system. The apparatus was comprised of an EG\&G model 283 potentiostat/galvanostat and an EG\&G model 1025 frequency response analyzer. The system was operated under computer control using an EIS software package from EG\&G. A solution of $0.1 \mathrm{M}$ of PBS buffer ( $\mathrm{pH}$ $=7$ ) was used as the electrolyte in a three-electrode cell. A platinum foil was used as the counter electrode, a saturated calomel electrode (SCE) was used as the reference electrode, and the probe sites, with or without polymer coatings, were used as the working electrode. An AC sinusoid with $5 \mathrm{mV}$ of amplitude was used as the input signal with the DC potential set to $0 \mathrm{~V}$. The value of the impedance was determined at five discrete frequencies per decade over the range of 10 to $10^{5} \mathrm{~Hz}$.

\section{Cyclic voltammetry}

Cyclic voltammetry (CV) was performed using a locally designed potentiostat combined with a phase lock amplifier (Stanford Research System SR 830), personal computer (Gateway 486 DX2/50), multiplexer (National Instruments SC-2062), and Labview software (National Instruments). The three-electrode cell setup was the same as that used in the impedance spectroscopy measurement. A scan rate of 100 $\mathrm{mV} / \mathrm{s}$ was used, and the potential on the working electrode was swept between -0.9 to $0.5 \mathrm{~V}$ versus SCE. This limit was wide enough to include the reversible redox reaction yet narrow enough to avoid overoxidation and remain within the water window. Before each $\mathrm{CV}$ was recorded, several cycles were swept to insure that the film reached its stable state.

\section{Acute neural recording}

Acute experiments in a guinea pig were used to verify and characterize in vivo electrical functionality of the coated sites. $\mathrm{NIH}$ guidelines for the care and use of laboratory animals (NIH Publication \#85-23 Rev. 1985) were observed. The guinea pig initially was anesthetized with Ketamine and Xylazine, with supplemental injections given regularly to maintain an appropriate level of anesthesia. At no time was the animal allowed to awaken from the anesthesia. The animal was placed on a DC-powered heating pad to prevent a drop in core body temperature. The surgical procedure began with a midline incision on the head of approximately 1.5 inches. The skull was fixed with a high degree of stability to a rigid bar by a bolt embedded in dental acrylic and anchored to the dorsal cranium with stainless steel screws. A craniotomy was performed to expose the dorsal surface of the cerebellum, the dura was removed, and the probe was inserted. The brain then was covered with a warm agar solution to prevent desiccation and to reduce brain pulsations. The electrode was advanced until a neuron was recorded on the tip-most site. The electrode then was advanced in $10-\mu \mathrm{m}$ steps using a hydraulic microdrive so that each site would pass by the same neuron. Neural data were recorded using an in-house built pre-amp. At each depth, data were obtained using DataWave acquisition hardware and software. The data were analyzed off-line to find the neural spikes for each channel at each depth.

\section{Statistical analysis}

Data are reported as the mean \pm the standard error of the mean. Cell attachment was compared for statistical significance using a one-tail $t$ test analysis with $p<0.05$ reported as significant.

\section{In vitro cell test}

Before electrochemical deposition, all the probes were subjected to a base cleaning procedure to remove organic and metallic debris on the probes that potentially might be harmful to cells. The probes were cleaned for $5 \mathrm{~min}$ at $80^{\circ} \mathrm{C}$ in a base solution containing 1 part ammonium hydroxide, 1 part $30 \%$ hydrogen peroxide, and 5 parts deionized water. After electrochemical deposition of polypyrrole/ biomolecules, the probes were rinsed thoroughly with deionized water and then soaked in deionized water for at least $24 \mathrm{~h}$. Finally, the probes were placed in culture dishes and sterilized by exposure to ultraviolet light overnight in a laminar flow hood.

Rat glial cells (C6) were grown in F-12 nutrient mixture (Ham; Gibco) supplemented with 10\% horse serum, 5\% FBS and $0.5 \%$ gentamycin in a humidified incubator with $5 \%$ $\mathrm{CO}_{2}$. Human neuroblastoma (SH-SY5Y) cells ${ }^{30}$ (passage 75 80) were grown in Dulbecco's modified Eagle medium (DMEM; Gibco) with sodium pyruvate and supplemented 
with $10 \%$ fetal bovine serum (FBS) and 1\% penicillin/ streptomycin in a humidified incubator with $10 \% \mathrm{CO}_{2}$.

The cells were suspended and dissociated by incubating in $0.05 \%$ trypsin/ $0.53 \mathrm{mM}$ EDTA for $5 \mathrm{~min}$. The cells then were re-seeded on coated and uncoated probe surfaces for cell attachment and cell growth monitoring. The cells were stained as follows:

1. Rhodamine phalloidin (Molecular Probes) is a red fluorescent actin-binding stain. Cells were fixed in $2 \%$ paraformaldehyde, rinsed with phosphate-buffered saline (PBS), incubated $10 \mathrm{~min}$ in $0.3 \%$ Triton $\mathrm{X}-100$ to permeabilize cell membranes, rinsed with PBS, incubated with rhodamine phalloidin (1:100 in PBS) for 30-45 min, and, finally, rinsed with PBS.

2. Hoechst 33342 (Molecular Probes) is a blue fluorescent DNA-binding stain. Cells were fixed as above and stained with Hoechst 33342 diluted 1:10 5 in PBS for 20 min and rinsed with PBS.

A Zeiss Axiophot-2 fluorescence microscope (Carl Zeiss, Inc.; Thornwood, New York) was used for imaging cells on the silicon probe. The images were obtained using an Optronics RGB-cooled digital camera and collected as TIFF images. Bio-gel mounting media (Fisher Scientific) was dropped on and around the probe to stabilize it in the culture dish, and then a glass cover slip was placed over it.

\section{RESULTS AND DISCUSSION}

\section{Electrochemical deposition}

The optical micrograph shown in Figure 2 demonstrates the formation of polypyrrole/PSS on a multichannel silicon probe. The films on the gold electrode sites were black in color, which is characteristic of the oxidized state of the conducting polymer polypyrrole. The amount of material coated onto the probe was controlled precisely by varying the passage of charge. As the reaction proceeded, the film grew thicker and spread beyond the area of the gold surface.

The SEM micrograph in Figure 3 shows a closer view of the PPy/SLPF film that has grown on the probe. The evolution of the film started from a bare gold electrode site and grew gradually from 1 to 4 to $10 \mu \mathrm{C}$. The morphology of the film was much different from that of a PPy/PSS coating. The PPy/PSS has a nodular fractal surface. ${ }^{31}$ PPy/SLPF forms finger-like fibers that stick out of the gold surface and grow longer and thicker as more material is deposited. Further deposition results in a collapsing of the fibers into a more dense morphology. The intermediate "fuzzy" morphology of the electrode polymer coating should provide a larger interfacial area for promoting cellRGD peptide interactions and neural signal transport.

\section{Microfocusing FTIR}

The chemical composition of the PPy/biomolecule coatings on the electrode sites was determined using reflective microfocusing FTIR. Figure 4 shows the IR spectrum of polypyrrole/SLPF coating compared with pure SLPF and polypyrrole $/ \mathrm{ClO}_{4}^{-}$. Polypyrrole doped with $\mathrm{ClO}_{4}^{-}$had only a broad absorption band between 1500-1600 $\mathrm{cm}^{-1}$, which comes from the polypyrrole since $\mathrm{ClO}_{4}{ }^{-}$does not have absorption in this range. Proteins have characteristic absorptions at about 1650,1550, and $1450 \mathrm{~cm}^{-1}$, which are called Amide I, Amide II, and Amide III bands. ${ }^{32}$ The spectrum

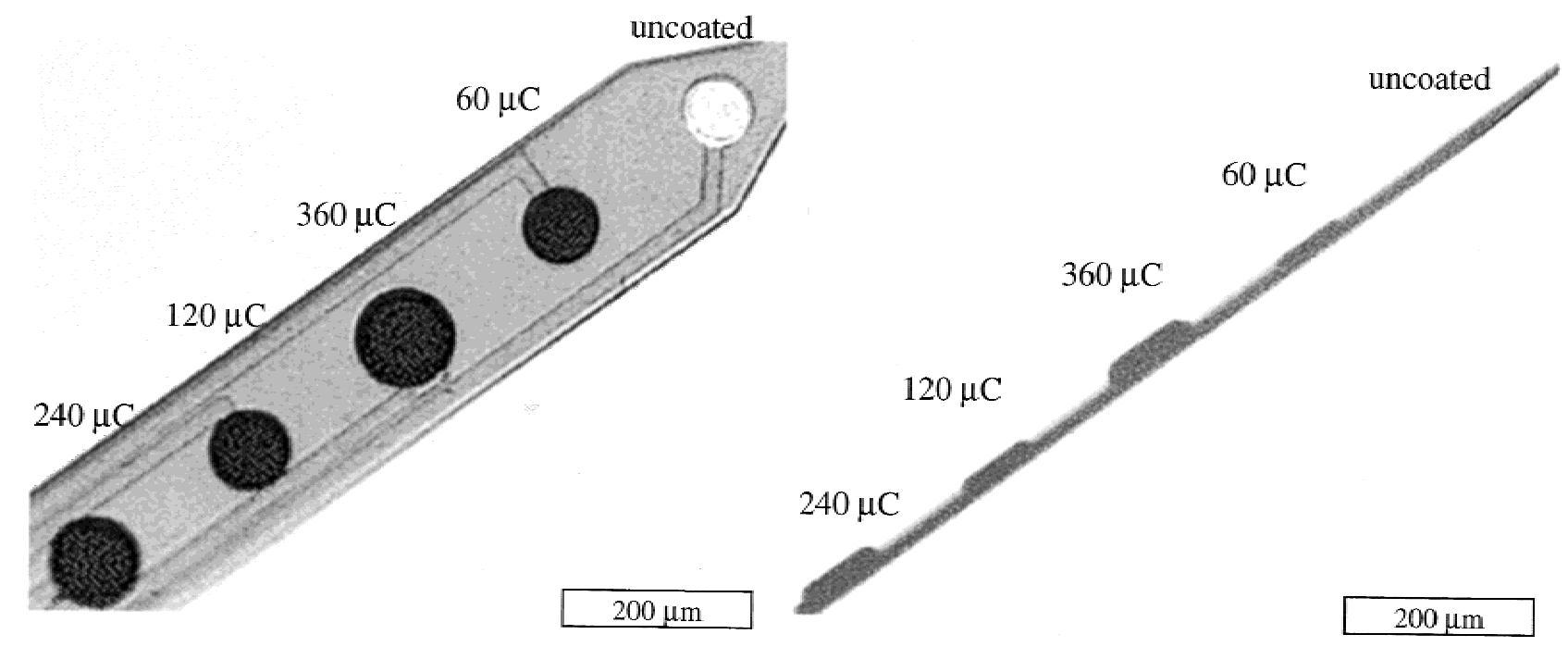

Figure 2. Optical micrographs of a polypyrrole/PSS-coated 5-channel neural probe. The left image is the top view of the probe, and the right image is the side view from which the thickness of the film can be estimated. The area of each metal electrode is $3900 \mu \mathrm{m}^{2}$, and the thickness of the probe is $15 \mu \mathrm{m}$. By varying the deposition charge, the amount of material deposited can be precisely controlled. 


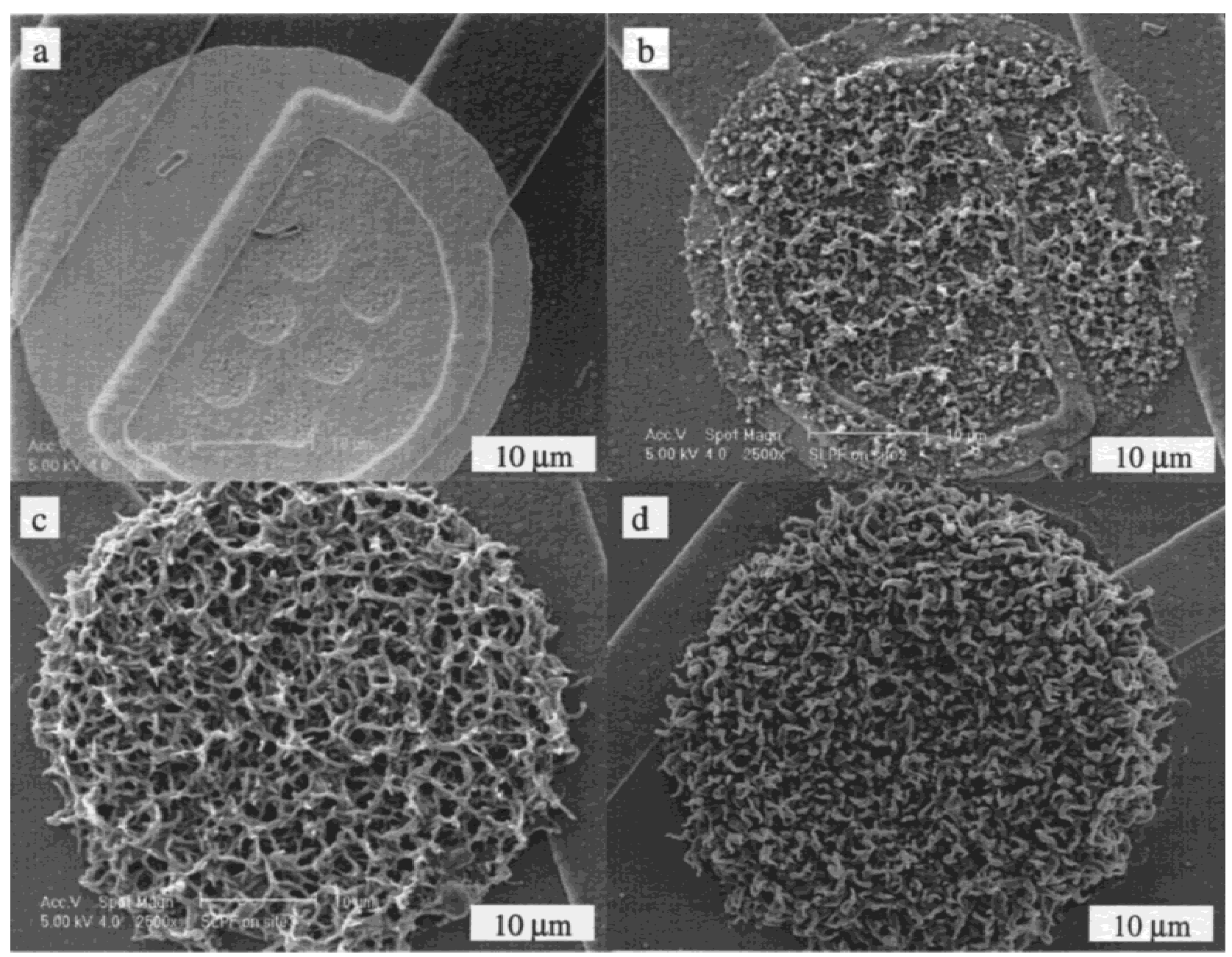

Figure 3. SEM images of PPy/SLPF-coated electrode sites. From (a) to (d) the deposition time increased, corresponding to a total charge passed of (a) $0 \mu \mathrm{C}$, (b) $1 \mu \mathrm{C}$, (c) $4 \mu \mathrm{C}$ and (d) $10 \mu \mathrm{C}$. The area of the uncoated electrode site is $1250 \mu \mathrm{m}^{2}$.

of pure SLPF showed three peak absorptions at 1650, 1547, and 1452, which can be assigned as Amide I, Amide II, and Amide III. The spectrum of PPy doped with SLPF was a combination of these two components. The three peaks at 1663, 1550, and 1452 correspond to the Amide I, Amide II, and Amide III, with the Amide II superimposed on the broad absorption band of pyrrole. This result confirms the existence of protein polymer in the coating. The Amide I peak positions shift $13 \mathrm{~cm}^{-1}$ from the pure SLPF to that incorporated in the conducting polymer, which might be due to subtle conformation changes caused by the interaction between polypyrrole backbone and the incorporated SLPF. Similarly, the incorporation of the peptide fragment CDPGYIGSR also was confirmed.

\section{Electronic properties}

The electronic and electrochemical properties of PPy/biomolecules were measured using electro-

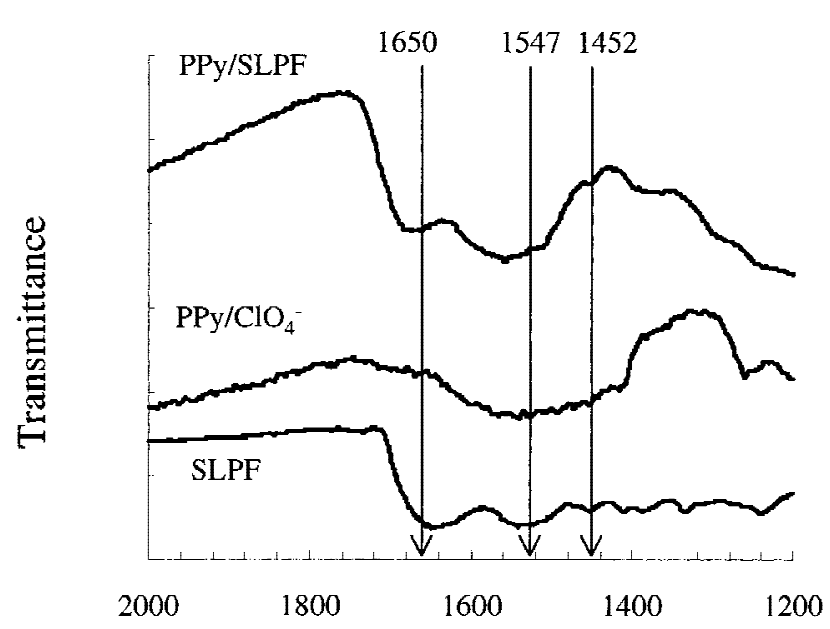

Wavenumber $\left(\mathrm{cm}^{-1}\right)$

Figure 4. Microfocusing FT-IR spectrum of polypyrrole/ SLPF coating compared to pure SLPF powder and polypyrrole $/ \mathrm{CIO}_{4}{ }^{-}$. 
chemical impedance spectroscopy (EIS) and cyclic voltammetry $(\mathrm{CV})$. Impedance is a complex quantity defined as $Z(\omega) \equiv|Z(\omega)| \mathrm{e}^{\mathrm{i} Z Z(\omega)} \equiv \mathrm{v}(\omega) / \mathrm{i}(\omega)$, where $\mathrm{v}(\omega)$ is the transform of an alternating potential signal applied to the system and $i(\omega)$ is the transform of the alternating current signal that results. The impedance is characterized by a magnitude $(|\mathrm{Z}|)$ and phase angle $(\theta)$, and most generally is represented as a function of frequency. For a capacitor, the impedance is purely imaginary, the current is out of phase with the voltage by $90^{\circ}$, and the phase angle is $90^{\circ}$. For a resistor, the impedance is real, the current and voltage are in phase, and the phase angle is $0^{\circ}$. For a system composed of some combination of these two components, a large phase angle value indicates that the impedance is predominantly capacitive while small-angle values are resistive. ${ }^{33}$

Impedance spectroscopy involves measuring the electrode impedance over a spectrum of frequencies. Information about the system under test is obtained by comparing the input signal to the output signal. Magnitude and phase information are measured directly. Using these data, one can get qualitative and quantitative information about the electrical properties of the system over a large range of frequencies as well as the morphology of the electrode-medium interface. Figure 5 shows the impedance spectra of polypyrrole/PSS- and polypyrrole/SLPF-coated electrode sites with an uncoated gold electrode spectrum as a control. After being coated with conducting polymer polypyrrole/PSS, the impedance across the whole frequency range was 1 2 orders of magnitude lower than the bare gold electrode [Fig. 5(a)]. This can be explained by the high conductivity of the polymer and, more important, by the increased interfacial area.

For PPy doped with SLPF-SO ${ }_{3}^{-}$, the impedance was higher in the high frequency region, in some cases even higher than the bare gold, but it was still lower in the lower frequency region from $10^{4}$ to $10 \mathrm{~Hz}$. The magnitude was low at $1 \mathrm{kHz}$, which is in the midrange of the biologic relevant frequency of neural activity. The phase plot of the impedance spectroscopy [Fig. 5(b)] showed that the phase was around $80^{\circ}$ for gold, which means the electrode was close to a pure capacitor. After coating with polypyrrole, the phase decreased to $\sim 50^{\circ}$ for PPy/PSS and $\sim 20^{\circ}$ for PPy/ $\mathrm{SLPF}$, which was an indication of a more resistive electrode.

From the Nyquist plot of impedance spectroscopy [Fig. 5(c)], which plots the imaginary part of the impedance (ImZ) versus the real part (ReZ), it is possible

Figure 5. Impedance spectroscopy of gold, PPy/SLPF coated and PPy/PSS coated electrodes. The uncoated gold electrode area is $3900 \mu \mathrm{m}^{2}$. (a) Magnitude of impedance as a function of frequency, (b) Phase of impedance as a function of frequency, (c) Nyquist plot. to assign values to the parameters of the circuit model

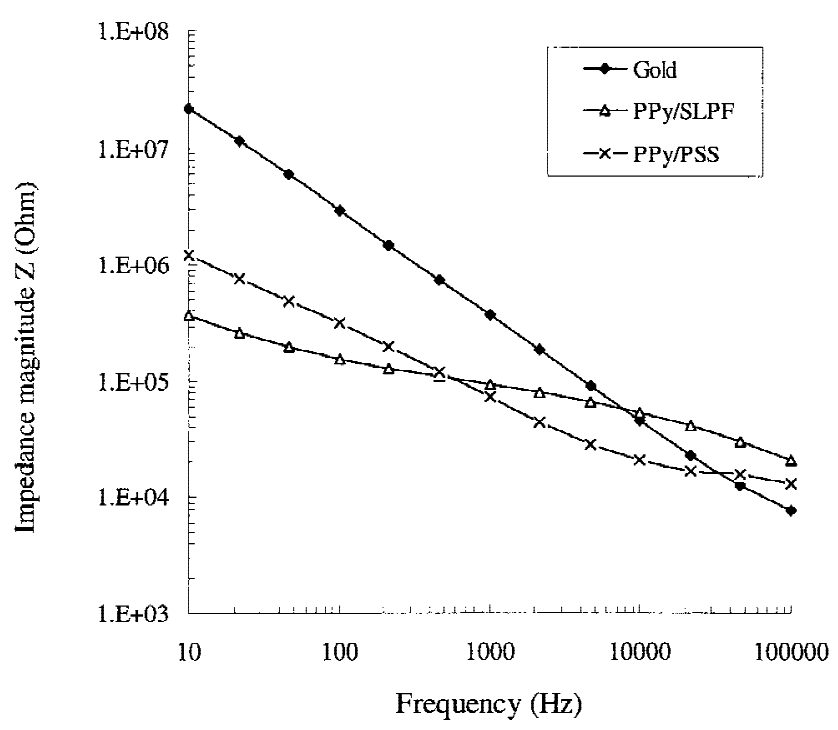

(a)

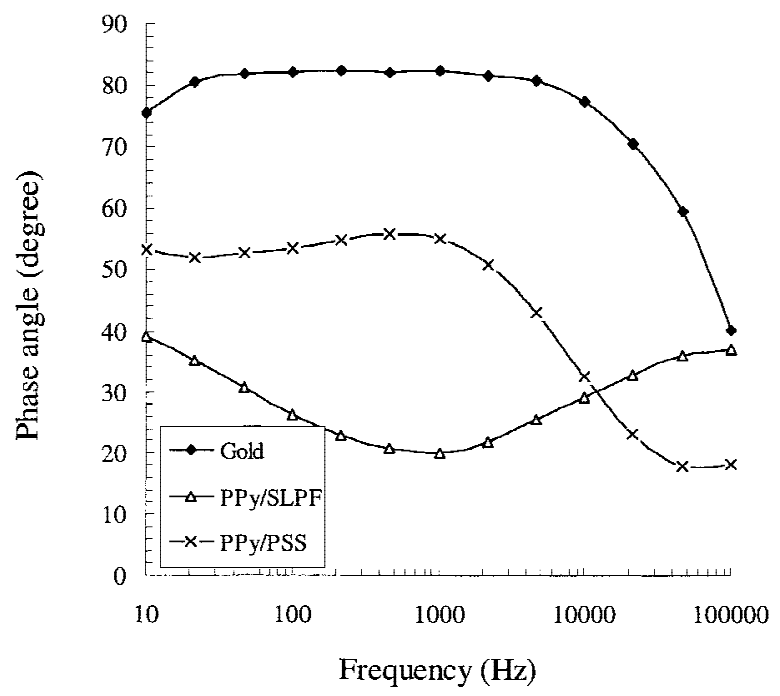

(b)

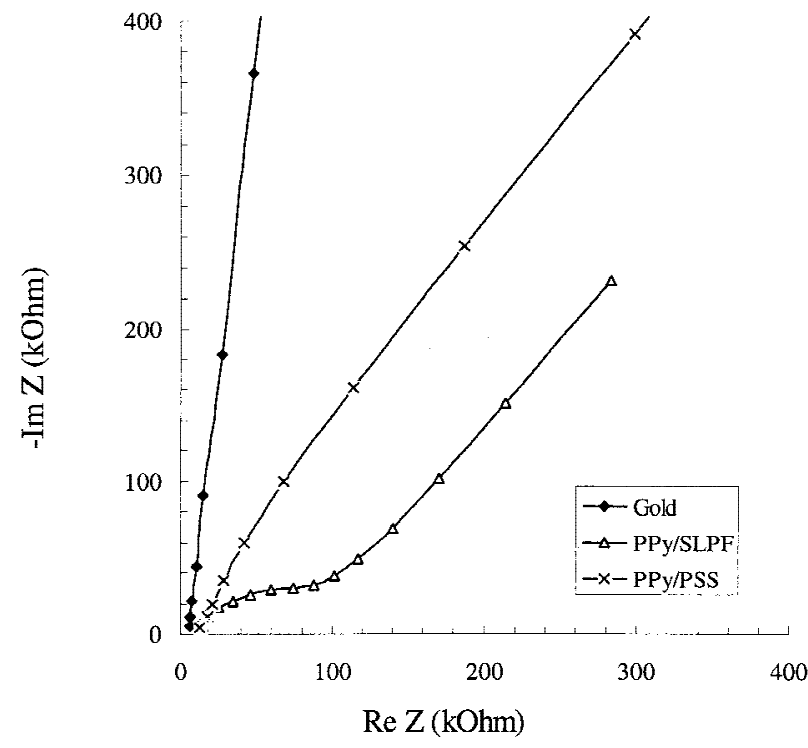

(c) 
of the system. ${ }^{34}$ While detailed circuit modeling on our system has not been performed, some generally accepted characteristics of model components describe the system qualitatively. The steep straight line of gold is typical of a pure capacitor. After coating with conducting polymer PPy/PSS, the slope of the straight line dropped to 1 . This is close to the Warburg impedance, which shows the frequency dependence of the impedance on mass transfer of ions to the interface. Typical Warburg impedance has a constant phase of $45^{\circ}$ while the trajectory of the plot of PPy/ PSS was not straight over the whole range and is higher than $45^{\circ}$ in the high frequency region. This probably can be explained by the rough, porous surface of the conducting polymer, which adds complexity and nonuniformity to the frequency-dependent impedance. $^{34,35}$

The Nyquist plot of PPy/SLPF exhibited a semicircle at the higher frequency portion as well as a straight-line trajectory at lower frequency portion. The semicircle indicates that an interfacial charge transfer process has become slow enough to be observed. The triple of the difference between the two intercepts of the semicircle with the ReZ axis gives an estimate of the ionic resistance of the film. ${ }^{36}$ In the PPy/PSS film, this part of the resistance is insignificant. The results indicate that the SLPF-doped PPy was not as electroconductive as PPy doped with PSS. This is not surprising considering the higher doping level of PPy/ PSS $^{-}$shown by the CV (as shown later) and the chemical chain structure of PSS $^{-}$, which was proposed to facilitate the interchain charge transport in the polymer. ${ }^{37}$

Impedance spectroscopy of polypyrrole/SLPF films deposited on gold electrodes varied nonlinearly as the amount of the material on the electrode changed. Figure 6 shows the variation of impedance magnitude at $1 \mathrm{kHz}$ as a function of deposition charge. A small amount of PPy/SLPF on the electrode increased the impedance one to two orders of magnitude higher than that of an uncoated gold site. There also was a significant impedance increase for the thickest films. Only an intermediate amount of material coated on the electrode gave the lowest impedance at $1 \mathrm{kHz}$. This optimum amount of material seems to correspond to the most fuzzy, fibrous morphology of the coatings, which provides the highest interfacial area for charge transport. The cartoon inserted in the figure schematically illustrates the correlation between the morphology and the signal transport across the interface.

There was a reversible redox reaction in the polypyrrole film. The redox reaction involves the charging and discharging of polypyrrole and also is accompanied by movement of ions. Because the oxidized state and reduced state have very different electrical properties, this redox reaction provides a switching ability

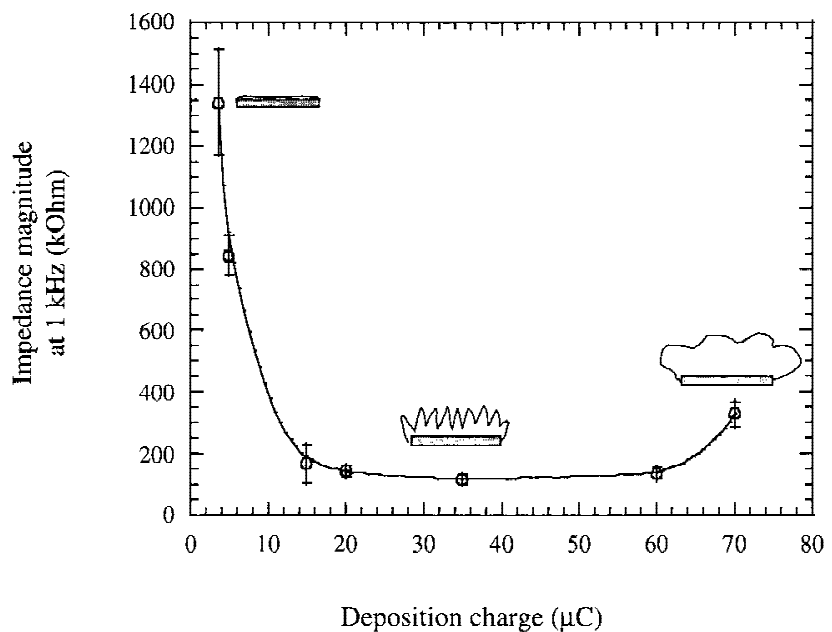

Figure 6. Impedance magnitude of PPy/SLPF films as a function of deposition charges. The cartoons show the coating morphology at three thicknesses. As the film starts to grow, it formed a very smooth thin layer on the electrode. The roughness of the film increased as the film grew thicker. The most hairy morphology resulted in a dramatic decrease in impedance. When the film was too thick, the impedance started to increase as a result of the decrease in surface area, detachment of film from electrode and increase of the water content. Data are reported as the mean \pm the standard error of the mean, and $n=3$.

to the doped polypyrrole films. Cyclic voltammetry shows the intrinsic redox reaction of the electrode material as the potential of the electrode is swept in a cyclic manner. As shown in Figure 7, no redox reactions occur at the gold electrode, but the switching

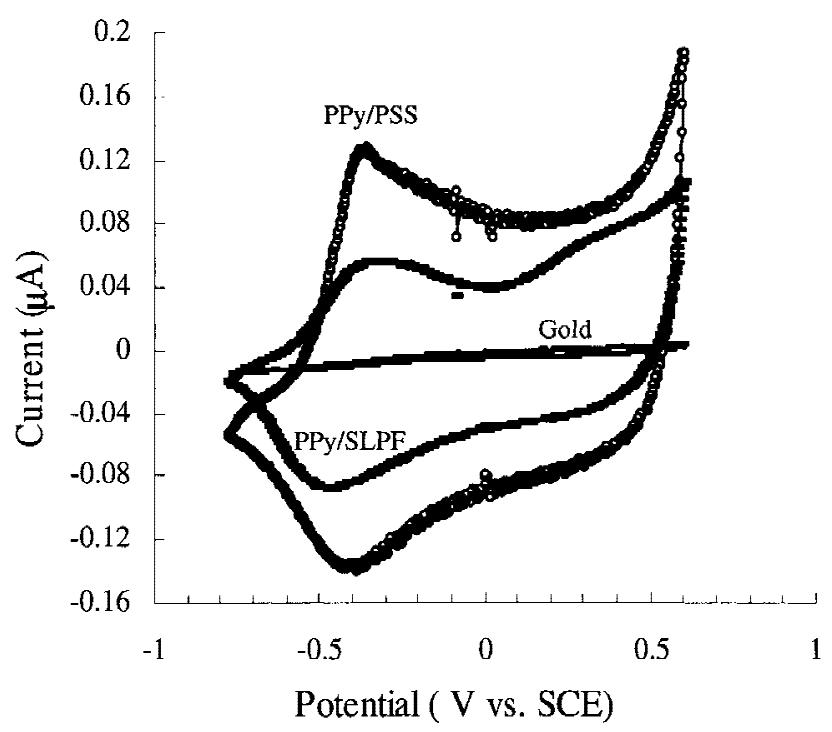

Figure 7. Cyclic voltammetry of polypyrrole/SLPF coated electrode in comparison with bare gold and PPy/PSS coated electrode. The electrode area is $1250 \mathrm{\mu m}^{2}$. PPy/PSS and PPy/SLPF were deposited on the gold electrode by passing $10 \mu \mathrm{C}$ charge at a current density of $0.5 \mathrm{~mA} / \mathrm{cm}^{2}$. The charge capacity calculated was $0.12 \mu \mathrm{C}$ for gold, $1.40 \mu \mathrm{C}$ for PPy/ SLPF, $2.33 \mu \mathrm{C}$ for PPy/PSS. 
ability of polypyrrole was demonstrated. Within a cycle of voltage sweeping, there are two peaks (one anodic and one cathodic) at potentials of -0.34 and $-0.52 \mathrm{~V}$ versus SCE, which are indications of oxidation and a reduction reaction of polypyrrole/PSS, respectively. The redox reaction was accompanied by the movement of small cations in and out of the film. Additionally, the interaction between the polypyrrole and the large dopant molecules were changed upon each reaction, as were the electronic properties of the film. This intrinsic switching property potentially could be exploited in a number of applications, such as ion exchanging, biosensing, and controlled release. ${ }^{38-40}$

The CV of PPy/SLPF also showed two redox peaks at approximately the same positions as those of PPy/ PSS. What was different in this case was the charge capacity. Since the voltage was swept at a constant scanning rate $(100 \mathrm{mV} / \mathrm{s})$, the curve of I (current) V (potential) could be converted linearly into I(t). The integration of I ( $t$ ) within the cycled region gave the charge capacity of the film, which was determined by how many mobile charges were moved in and out of the film during the cycle. The number of mobile charges was determined by several factors. The first factor was the doping level, or oxidation degree of the polypyrrole. The higher the doping level, the more charges were available to move. The second factor was the interfacial area between the coated electrode and the electrolyte solution. A higher interfacial area provides more sites with which ions can interact. Therefore the morphology of the film plays an important role. At the same doping level, one expects that a very dense and smooth electrode surface would have a lower charge capacity than would a porous and hairy surface. For PPy/SLPF and PPy/PSS ${ }^{-}$synthesized using the same electrochemical conditions (same current density and charge passage), the former showed a lower charge capacity, which was due mainly to the lower doping level. This was not difficult to explain considering the low ion density of the SLPF compared to that of the $\mathrm{PSS}^{-}$.

To insure that the organic film coated on the electrode did not interfere with the recording process of the neural probes, acute in vivo recording tests were conducted at the Kresge Hearing Research Institute. Figure 8 shows a diagram of the recording electrode next to sample recordings for four depths. The probe senses an individual neuron at a given electrode site, and as the probe was advanced further into tissue, the response passed from one electrode site to another. Channel 5 is the tip-most site while channel 7 is the top-most site. The results confirmed that high quality neural signals could be recorded from single neurons in the cerebellum of guinea pig through both coated and uncoated electrodes.
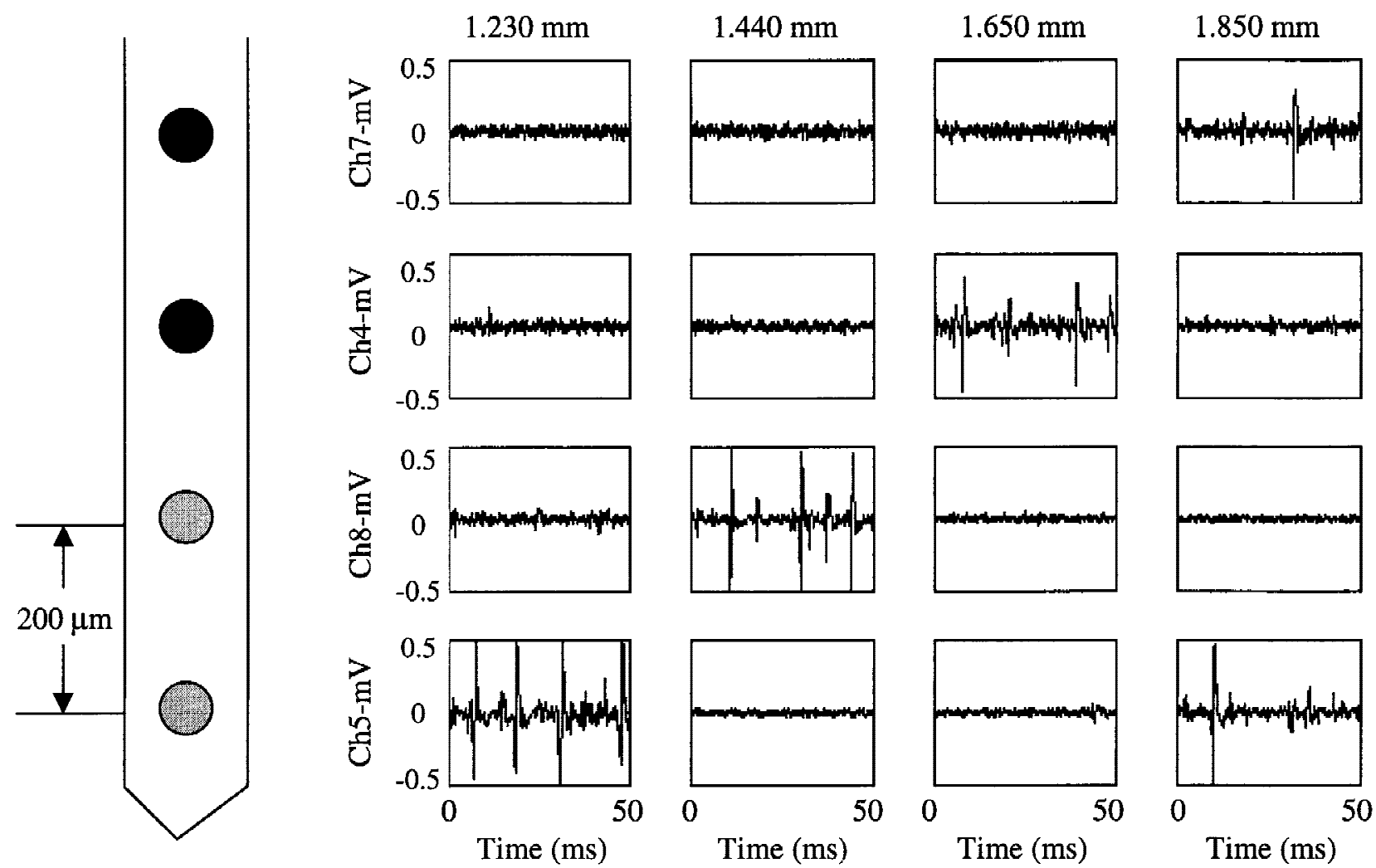

Figure 8. Acute recording test from cerebellum of guinea pig. Channel 5 and 8, Au, uncoated; Channel 4 and 7, PPy/SLPF coated. The probe was advanced in $10 \mu \mathrm{m}$ steps, so that each recording site passed the same neuron. 


\section{In vitro cell test}

Silicon probes with coated and uncoated electrode sites were cultured with rat glial and human neuroblastoma cells for different amounts of time. Both types of cells grew and proliferated normally in the presence of the silicon probe and conducting polymer blends. This indicates that after the cleaning procedure, the silicon probe and coatings were not harmful to cells in vitro. To compare the response of cells to the coated and uncoated electrode sites, polypyrrole/ SLPF was coated on eight active sites of a 4-shank, 16-channel microelectrode array. The black spots were coated electrode and the bright spots were bare gold electrodes (Fig. 9). It was possible to study different samples (coated and uncoated in this case) in the same culture dish. The rat glial cells were seeded and cultured for $24 \mathrm{~h}$, which was long enough for cells to attach tightly onto the surface and short enough to exclude the possibility of proliferation. It was clearly shown that cells selectively attached on the polypyrrole/SLPF-coated electrode. The DNA of the cells was stained by DNA probe so that each bright spot in the image corresponded to one nucleus. According to a statistical analysis, there were significantly $(p<0.05)$ more cells $(1.25 \pm 0.6$ cells per site $)$ on PPy/SLPF-

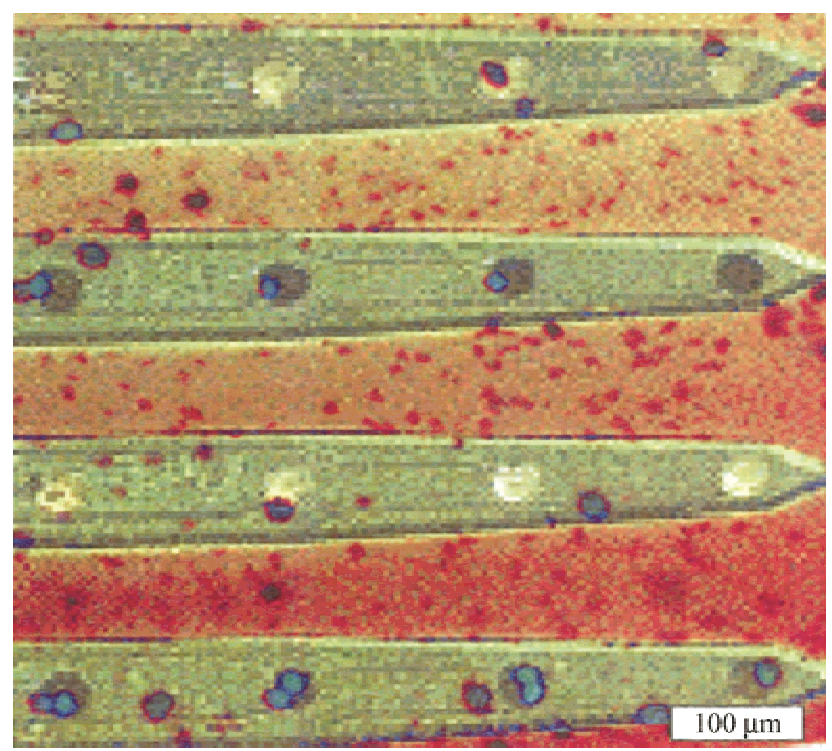

Figure 9. PPy/SLPF coated 4-shank 16-channel neural probe cultured with rat glial cells. The dark black spots are coated electrode sites and the bright ones are uncoated. The optical micrograph of the probe before culturing and the fluorescence micrograph of the probe after culturing and staining were integrated in Adobe Photoshop 5.0, in order to show both the pattern of the electrode sites and the cells on the probe. Cells were stained using Hoechst 33342, and the blue spots correspond to individual cells. Significantly more cells attach to the coated electrode sites than the uncoated areas. [Color figure can be viewed in the online issue, which is available at www.interscience.wiley.com.]

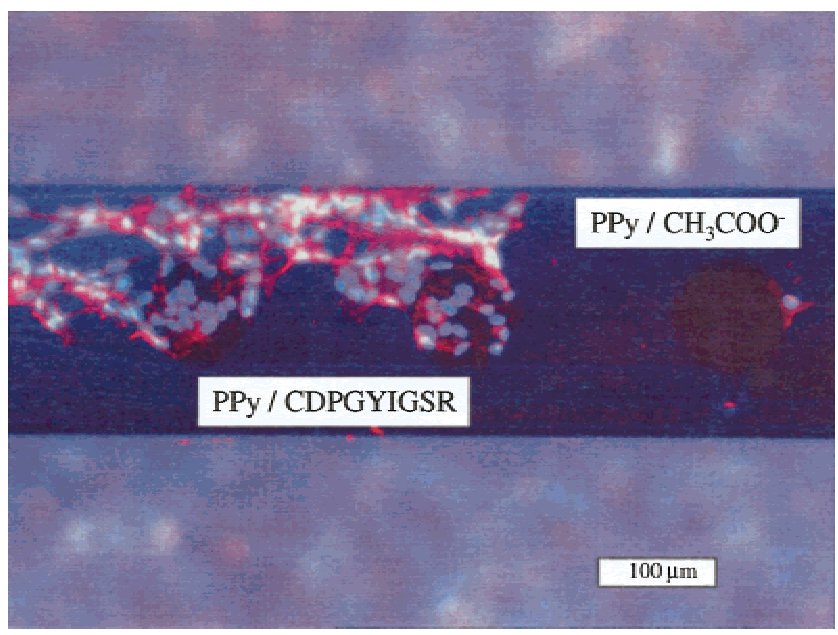

Figure 10. Coated neural probe cultured with human neuroblastoma cells. Different materials were coated on the electrode as indicated in the figure. To help visualize the cells on the probe, fluorescence molecular probe Hoechst 33342 was used to stain the DNA (blue) and Rhodamine Phalloidin was used to stain the actin filaments (red). Two images using blue and red fluorescent light were taken respectively and integrated into one single image in Adobe Photoshop 5.0. [Color figure can be viewed in the online issue, which is available at www.interscience.wiley.com.]

coated electrode sites than there were on uncoated gold electrode $(0.25 \pm 0.4)$.

PPy/CDPGYIGSR and PPy/CH3COO${ }^{-}$were coated on a 4-channel single-shank probe. The probe was cultured with human neuroblastoma cells for 3 days, and the results can be seen in Figure 10. The neuroblastoma cells selectively seeded onto the CDPGYIGSRcontaining electrode, spread beyond the electrode area, and formed a network between two coated electrodes. The electrode coated with $\mathrm{PPy} / \mathrm{CH}_{3} \mathrm{COO}^{-}$, which does not have cell-binding species, showed no preferential attraction to cells.

With the combination of silicon-based microelectronic fabrication technology and electrochemical deposition of conducting polymer/biomolecule blends, different biochemical species can be patterned onto a small device, and, subsequently, the distribution of cells with high spatial resolution can be controlled. More important, cells are selectively attracted to electrode sites, which should help to improve the communication between these cells and the recording or stimulating system. By adding different biologic molecules to site coatings, we expect to be able to attract different cells onto certain sites of the same electrode array and to record the activity of cells through different channels. It also may be possible to create neural networks on such a device to develop and research neural communication in vitro. After immobilization of cells in vitro, the device could be put into the living system to function as a neural prosthesis. 


\section{CONCLUSIONS}

The deposition of conducting polymer/biomolecule blends on silicon microelectrodes was performed precisely and reproducibly. The thickness and morphology of the coatings was adjusted by controlling the reaction conditions. A convex, "fuzzy" morphology of a coating seemed to be better than a flat surface for establishing an intimate interfacial contact with tissue. The coated electrode had a much higher surface area and charge capacity, which provided a larger efficient interface for electronic and ionic signal transport. More important, biomolecules having cell-binding functionality were incorporated into the coating. Preferred cell attachments on the coated electrodes were demonstrated in vitro. This approach should make it possible to attract and anchor cells on the electrode in vivo and thus to obtain a more stable and long-lasting recording device. Future work will involve the assessment of coatings on neural probes in chronic implantation. Cellular response to the coating and neural activity recording through coated electrodes will be evaluated to test the above hypothesis.

Dr. Richard Altschuler in the Department of Otolaryngology and the Kresge Hearing Research Institute of the University of Michigan provided materials, instruments, and technical support. Dr. Joseph Cappello of Protein Polymer Technologies, Inc. provided the protein polymer and information about the solubilization method.

\section{References}

1. Niparko JK, Altschuler RA, Xue X, Wiler JA, Anderson DJ. Surgical implantation and biocompatibility of central nervous system auditory prostheses. Ann Oto Rhinol Laryngol 1989;98: 965-970.

2. Oweiss K, Wise M, Lopez C, Wiler JA, Anderson D. Chronic electrode-brain interface modeled with FEM. Proc First Joint BMES/EMBS Conf Serving Humanity, Adv Technol, Oct 1316, Atlanta; 1999.

3. Turner JN, Shain W, Szarowski DH, Andersen M, Martins S, Isaacson M, Craighead $\hat{\mathrm{E} H}$. Cerebral astrocyte response to micromachined silicon implants. Exp Neurol 1999;156:3349.

4. Buchko CJ. Processing and characterization of protein polymer thin films for surface modification of neural prosthetic devices [PhD Thesis]. Michigan: The University of Michigan; 1997.

5. Mensinger AF, Anderson DJ, Martin D, Buchko CJ, Johnson MA, Martin DC, Tresco PA, Silver RB, Highstein SM. Chronic recording of VIIIth nerve axons with a sieve electrode. J Neurophysiol 2000;83:611-615.

6. Schmidt CE, Shastri VR, Vacanti JP, Langer R. Stimulation of neurite outgrowth using an electrically conducting polymer. Proc Natl Acad Sci USA 1997;94:8948-8953.

7. Wallace GG. Conductive electroactive polymers: Intelligent materials systems. Lancaster, Pennsylvania: Technomic Publishing Co.; 1997.

8. Silk T, Hong Q, Tamm J, Compton RG. AFM studies of poly- pyrrole film surface morphology. I. The influence of film thickness and dopant nature. Syn Metals 1998;93:59-64

9. Wang J, Jiang M. Toward genolelectronics: Nucleic acid doped conducting polymers. Langmuir 2000;16:2269-2274.

10. Garner B, Georgevich A, Hodgson A, Liu L, Wallace GG. Polypyrrole-heparin composites as stimulus-responsive substrate for endothelial cell growth. J Biomed Mater Res 1999;44:121129.

11. Freemantle M. The endless polymer science frontier. Chem Eng News 2000;78:39-45.

12. Hodgson AJ, John MJ, Campbell T, Georgevich A, Woodhouse S, Aoki T, Ogata N, Wallace GG. Integration of biocomponents with synthetic structures-Use of conducting polymer polyelectrolyte composites. SPIE 1996;2716:164-176.

13. Martin GR, Timpl R. Laminin and other basement-membrane components. Ann Rev Cell Biol 1987;3:57-85.

14. Kleinman HK, Cannon FB, Laurie GW, Hassell JR, Aumailley M, Terranova VP, Martin GR, Dubois-Dalcq M. Biological activities of laminin. J Cell Biochem 1987;27;317-325

15. Thanos S, Bahr M, Barde YA, Vanselow J. Survival and axonal elongation of adult rat retinal ganglion cells: In vitro effects of lesioned sciatic-nerve and brain-derived neurotrophic factor. Eur J Neurosci 1989;1:19-26.

16. Grant DS, Tashiro KI, Segulreal B, Yamada Y, Martin GR, Kleinman HK. Two different laminin domains mediate the differentiation of human-endothelial cells into capillary-like structures in vitro. Cell 1989;58:933-943.

17. Nomizu M, Weeks BS, Weston CA, Kim WH, Kleinman HK, Yamada Y. Structure-activity study of a laminin $\alpha 1$ chain active peptide segment Ile-Lys-Val-Ala-Val (IKVAV). FEBS Lett 1995; 365:227-231.

18. Graf J, Ogle RC, Robey FA, Sasaki M, Martin GR, Yamada Y, Kleinman HK. A pentapeptide from the laminin-B1 chain mediates cell adhesion and binds the 67000-laminin receptor. Biochemistry 1987;26:6896-6900.

19. Graf J, Iwamoto Y, Sasaki M, Martin GR, Kleinman HK, Robey FA, Yamada Y. Identification of an amino-acid sequence in laminin-mediating cell attachment, chemotaxis, and receptor binding. Cell 1987;48:989-996.

20. Borkenhagen M, Clemence JF, Sigrist H, Aebischer P. Threedimensional extracellular matrix engineering in the nervous system. J Biomed Mater Res 1998;40:392-400.

21. Massia SP, Rao SS, Hubbell JA. Covalently immobilized laminin peptide Tyr-Ile-Gly-Ser-Arg (YIGSR) supports cell spreading and co-localization of the 67-kilodalton laminin receptor with $\alpha$-actinin and vinculin. J Biol Chem 1993;268:8053-8059.

22. Boeckl MS, Baas T, Fujita A, Hwang KO, Bramblett AL, Ratner BD, Rogers JW, Sasaki T. Template-assisted nano-patterning of solid surfaces. Biopolymer 1998;47:185-193.

23. Pakalns T, Haverstick KL, Fields GB, McCarthy JB, Mooradian DL, Tirrell M. Cellular recognition of synthetic peptide amphiphiles in self-assembled monolayer films. Biomaterials 1999;20: 2265-2279.

24. James CD, Davis RC, Kam L, Craighead HG, Isaacson M, Turner JN, Shain W. Patterned protein layers on solid substrates by thin stamp microcontact printing. Langmuir 1998;14: 741-744.

25. Huber M, Heiduschka P, Kienle S, Pavlidis C, Mack J, Walk T, Jung G, Thanos S. Modification of glassy carbon surfaces with synthetic laminin-derived peptides for nerve cell attachment and neurite growth. J Biomed Mater Res 1998;41:278-288.

26. Cappello J, Crissman JW. Design and production of bioactive protein polymers for biomedical applications. Abstract. Am Chem Soc 1990;199:66.

27. Drake KL, Wise KD, Farraye J, Anderson DJ, and BeMent SL. Performance of planar multisite microprobes in recording extracellular single-unit intracortical activity. IEEE Trans Biomed Eng 1988;35:719-732. 
28. Anderson DJ, Najafi K, Tanghe SJ, Evans DA, Levy KL, Hetke JF, Xue X, Zappia JJ, Wise KD. Batch-fabricated thin-film electrodes for stimulation of the central auditory system. IEEE Trans Biomed Eng 1989;36:693-704.

29. Stedronsky ER. Chemical modification of repetitive polymers to enhance water solubility. US patent no. 5,760,004; 1994.

30. Sonnenfeld KH, Ishii DN. Nerve growth factor effects and receptors in cultured human neuroblastoma cell lines. J Neurosci Res 1982;8:375-391.

31. Silk T, Hong Q, Tamm J, Compton RG. AFM studies of polypyrrole film surface morphology. II. Roughness characterization by the fractal dimension analysis. Syn Metals 1998;93:65-71.

32. Byler DM, Susi H. Examination of the secondary structure of proteins by deconvolved FTIR spectra. Biopolymers 1986;25:469-487.

33. MacDonald JR, editor. Impedance spectroscopy: Emphasizing solid materials and systems. New York: John Wiley \& Sons;1987.

34. Komura T, Goisihara S, Yamaguti T, Takahasi K. Electron and ion transport in polypyrrole/polystyrenesulfonate composite films. J Electroanal Chem 1998;456:121-129.
35. Weiland JD, Anderson DJ. Chronic neural stimulation with thin-film, iridium oxide electrode. IEEE Trans Biomed Eng 2000;47:911-918.

36. Ren X, Pickup PG. An impedance study of electron transport and electron transfer in composite polypyrrole + polystyrenesulphonate films. J Electroanal Chem 1997;420:251-257.

37. Bhattacharya A, De A, Das S. Electrochemical preparation and study of transport properties of polypyrrole doped with unsaturated organic sulfonates. Polymer 1996;37:4375-4382.

38. Miller LL, Zhou QX. Poly(N-methylpyrrolylium) poly(styrenesulfonate). A conductive, electrically switchable cation exchanger that cathodically binds and anodically releases dopamine. Macromolecules 1987;20:1594-1597.

39. Pernaut JM, Reynolds JR. Use of conducting electroactive polymers for drug delivery and sensing of bioactive molecules. A redox chemistry approach. J Phys Chem B 2000;104:4080-4090.

40. Kontturi K, Pentti P, Sundholm G. Polypyrrole as a model membrane for drug delivery. J Electroanal Chem 1998;453:231238. 